\title{
Gastric mucosal contraction and vascular injury induced by indomethacin precede neutrophil infiltration in the rat
}

\author{
A Anthony, R Sim, A P Dhillon, R E Pounder, A J Wakefield
}

\begin{abstract}
Background-In contrast with earlier reports that neutrophils play a primary part in non-steroidal anti-inflammatory drug (NSAID) injury to the stomach, recent evidence suggests only a secondary role for these cells.

Aim-To examine whether early microscopic changes induced by indomethacin in the gastric corpus of fasted rats and the antrum of fasted-refed rats involve neutrophil infiltration.

Methods-Oral indomethacin $30 \mathrm{mg} / \mathrm{kg}$ or vehicle was given to six groups of fasted rats that were killed five, 15 , and 30 minutes after dosing. Subcutaneous indomethacin $30 \mathrm{mg} / \mathrm{kg}$ was also given to six groups of fasted-refed rats that were killed one, two, and four hours later. Haematoxylin and eosin and reticulin stained sections were examined to identify mucosal architectural changes. The gastric mucosa was also examined immunohistochemically for actin, fibrin, and neutrophils.
\end{abstract}

Results-In both the corpus and antrum, indomethacin caused an early phase of mucosal injury that occurred prior to mucosal neutrophil infiltration. Within the superficial corpus mucosa, this phase preceded coagulative necrosis and included surface epithelial expulsion, mucosal contraction with capillary aggregation and distortion, intravascular vascular fibrin deposition, and capillary congestion. The antrum showed similar early changes except that full thickness mucosal coagulative necrosis was a predominant early finding.

Conclusions-In two experimental models of NSAID gastric ulceration the mucosa undergoes early contraction, vascular fibrin deposition, and necrosis prior to neutrophil infiltration. These findings support a primary, neutrophil independent, ischaemic pathogenesis for NSAID gastric ulceration.

(Gut 1996; 39: 363-368)

Keywords: indomethacin, stomach, ulceration, neutrophils, contraction, mucosa.

Injury to the gastrointestinal tract limits the use of non-steroidal anti-inflammatory drugs (NSAIDs) in many rheumatology patients ${ }^{1}$ : consequently, much research effort has attempted to examine the mechanisms involved in mucosal damage. ${ }^{2}$ An important role for neutrophils in NSAID injury to the stomach has been suggested on the basis that antibodies against leucocyte endothelial adhesion molecules and depletion of circulating neutrophils in laboratory animals protected the mucosa. ${ }^{3-6}$ Wallace et al hypothesised that circulating neutrophils, in response to NSAID administration, occlude blood flow by adhering to the vascular endothelium and release tissue damaging free radicals and proteases. $^{7}$

However, recent studies have questioned the idea that NSAID injury to the gastrointestinal mucosa is neutrophil dependent. Arndt et al showed that while antibodies against CD11b/ CD18 and E-selectin reduced both indomethacin induced leucocyte-endothelial cell interactions within rat mesenteric venules and intestinal myeloperoxidase activity, the antibodies failed to prevent jejunal ulceration ${ }^{8}$; the authors concluded that granulocyte recruitment into the intestines is a consequence, rather than a cause of, mucosal lesions. In a histological study in rats, Whiteley et al showed that early indomethacin induced gastric erosions did not contain more neutrophils than vehicle control rats, suggesting that other mechanisms must be involved. ${ }^{9}$ Recently, Trevethick et al and Melarange et al showed that depletion of circulating neutrophils in the rat did not affect the extent of indomethacin induced gastrointestinal ulceration ${ }^{10}{ }^{11}$; only the number of neutrophils infiltrating the necrotic ulcer bed was severely reduced.

In a previous detailed histological study of the rat jejunum, we demonstrated that one hour after oral indomethacin, early features of mucosal ulceration included aggregation and clumping of villous smooth muscle, villous shortening, and distortion and thrombosis of the villous vasculature that occurred prior to significant neutrophil infiltration. ${ }^{12-14}$

In this paper we examined the corpus and antral mucosae for early neutrophil independent injury induced by indomethacin in fasted and fasted-refed rats.

\section{Methods}

Materials

Indomethacin was purchased from the Sigma Chemical Company (St Louis, MO, USA) and administered as a solution in $1.5 \%$ sodium bicarbonate ( $\mathrm{pH} \mathrm{8.4).} \mathrm{Male} \mathrm{Sprague-Dawley}$ 
rats $(240-270 \mathrm{~g})$ were supplied by $\mathrm{BK}$ Universal Ltd (Oldbrough, Hull, UK). Food was withheld from animals for no longer than 18 hours as described below but water was freely available at all times. During fasting the rats were kept in wire bottom cages to prevent coprophagy.

\section{Study design}

The study involved examination of the rat gastric mucosa using two models of indomethacin induced ulceration: corpus lesions were induced in fasted rats after oral indomethacin and antral lesions were induced in fasted-refed rats after subcutaneous indomethacin. ${ }^{15}$ Only corpus lesions are induced in fasted rats and only antral lesions are induced in fasted-refed rats. Twelve groups of rats were used.

Corpus ulceration in fasted rats

Six groups ( $n=6$ rats per group) were fasted overnight for 18 hours. In the morning, three groups received a single oral dose of indomethacin $30 \mathrm{mg} / \mathrm{kg}$ and were killed five, 15 , and 30 minutes later. Three control groups received vehicle only.

\section{Antral ulceration in fasted-refed rats}

The other six groups ( $n=5$ rats per group) were fasted overnight for 18 hours. They were then allowed free access to food for 60 minutes. Three groups then received a single subcutaneous dose of indomethacin $30 \mathrm{mg} / \mathrm{kg}$ and killed one, two, and six hours later. Three control groups received vehicle only. Rats were allowed continued access to food after dosing.

\section{Dosing and perfusion fixation of the small intestine}

At the end of the study period the rats were anaesthetised with $3.5 \%$ halothane and laparotomised through a mid-line incision. Heparin $(0 \cdot 1 \mathrm{ml}$ of $1000 \mathrm{U} / \mathrm{ml})$ was injected into the inferior vena cava and the animal was killed by removal of $5-10 \mathrm{ml}$ of blood by cardiac puncture after opening the thorax. The aorta was cannulated with $1.34 \mathrm{~mm}$ intravenous tubing and perfused with $10 \%$ formalin/normal saline at a mean arterial pressure of $80 \mathrm{~mm} \mathrm{Hg}$ for three minutes. The large vessels above the diaphragm were clamped and the portal vein was punctured to permit exit of perfusate. In this way, the entire gastrointestinal tract was perfusion fixed for optimal preservation of tissues. After overnight immersion fixation in formalin, the stomach was opened by cutting along the lesser and greater curvatures. The corpus and antrum was then examined with the unaided eye for visible lesions. Using a ruler, each lesion was scored by multiplying the maximum width and maximum length $\left(\mathrm{mm}^{2}\right)$. The total score for each rat was determined by summation of the lesion scores.
Histological and immunohistochemical analysis For each rat, most of the gastric corpus and antrum (about $80 \%$ of total) was cut into about 20 strips of tissue that were processed into paraffin wax and cut into $3 \mu \mathrm{m}$ sections. Serial sections from all tissues were stained with haematoxylin and eosin and reticulin (a silver stain that identifies vascular and epithelial and glandular basement membranes). Serial sections were also stained immunohistochemically with antibodies against smooth muscle $\alpha$ actin (to identify contractile cells, Dako), fibrinogen (which immunostains fibrinogen and early cross linked fibrin, Dako AS Glostrup, Denmark), and neutrophils (anti-neutrophil serum (ANS); Glaxo Research and Development, Ware, UK) as described previously. ${ }^{13}$ The avidin-biotin method was used with hydrogen peroxide and diaminobenzidine as the colorisation step.

\section{Morphological assessment}

Histological sections from the indomethacin dosed and control groups were examined for mucosal abnormalities by a 'blinded' observer. Within each histological lesion (one to eight lesions per indomethacin dosed rat) the total number of neutrophils were counted using the ANS stained sections and the mean neutrophil count/lesion was calculated for each rat. The mean number of neutrophils were also determined in the mucosal folds (six to eight per rat) of the vehicle control rats.

Using a calibrated eye piece graticule, the mucosal thicknesses of lesions maintaining an intact mucosa and neighbouring normal mucosa were measured and expressed as a ratio of the two values. Mucosal thickness was defined as the distance between the superficial margin of the muscularis mucosae and the surface capillaries. The mean ratio was determined for each rat (two to eight lesions per rat).

Where possible, photomicrographs of the edges of pathological lesions were taken so that representative areas of both pathological change and mucosal normality could be compared. The original magnification is given in the Figure legends.

\section{Results}

Gastric lesions in fasted rats

Indomethacin induced a macroscopic erosive gastritis of the corpus mucosa in six of six rats at 30 minutes, four of six of the rats at 15 minutes but in none of the rats at five minutes (Fig 1). The lesions were long and thin in appearance, black in coloration, and were usually sited along the crests or the sides of rugal folds. The antrum was normal in all indomethacin dosed rats. None of the control rats developed either corpus or antral lesions. The stomachs of rats from all indomethacin and control groups were empty of contents upon opening.

Although macroscopically normal at five minutes after dosing with indomethacin, the 


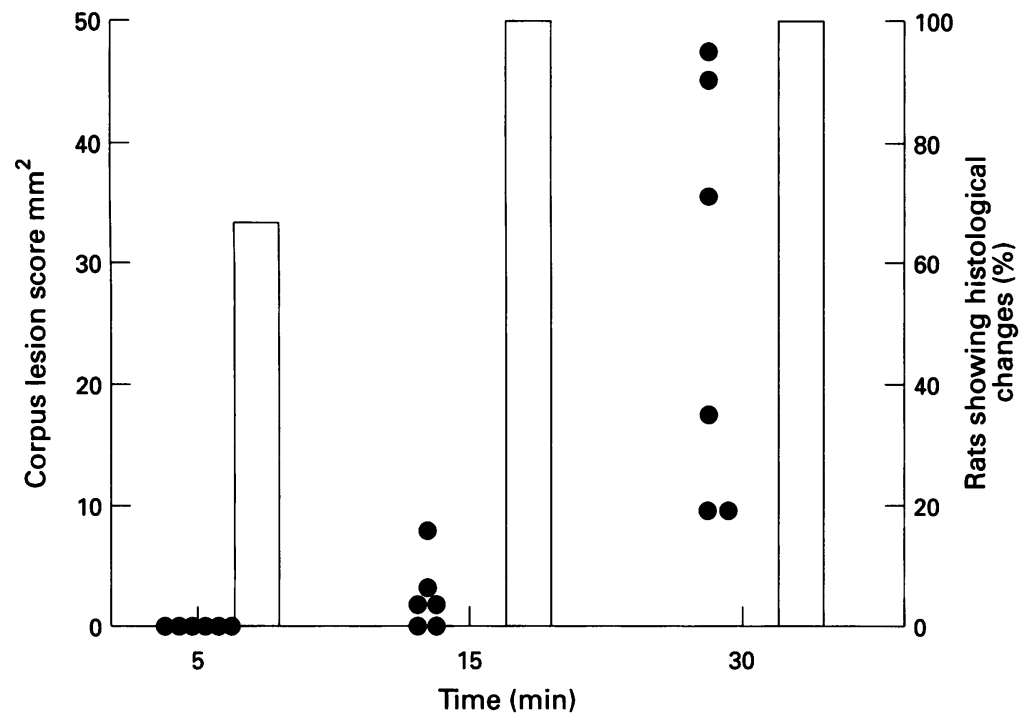

Figure 1: The extent of visible and histological injury induced by oral indomethacin $30 \mathrm{mg} / \mathrm{kg}$ in the corpus and antrum of fasted rats at five, 15, and 30 minutes after dosing. Filled circles $=$ corpus ulcer score for individual indomethacin dosed rats ( $n=6$ per group). Open bar=percentage of rats showing histological corpus changes. The antrum was macroscopically and histologically normal in all indomethacin dosed rats. None of the corresponding vehicle control rats showed any macroscopic or histological abnormality to either the corpus or antrum. corpus mucosa showed discrete histological abnormalities on the apex and sides of rugal folds in four of six rats (Fig 1). The superficial third of the mucosa appeared thinner than the normal adjacent mucosa with crowding of both the glandular cells and capillaries. The surface epithelium appeared to be extruded. These changes gave the upper mucosa a contracted appearance that was most apparent in the haematoxylin and eosin and reticulin stained sections (Figs 2 and 3; Table I). The actin stain for contractile cells showed increased staining and shortening and clumping of these mucosal elements. We think the increased actin staining within these cells reflects squeezing of the intracellular actin filaments during mucosal contraction. There was fibrin deposition within the surface capillaries. Neutrophils were notably absent from the mucosa, the rest of the stomach wall, and the mesentery (Table I).

At 15 minutes, mucosal contraction was more noticeable with epithelial loss of surface

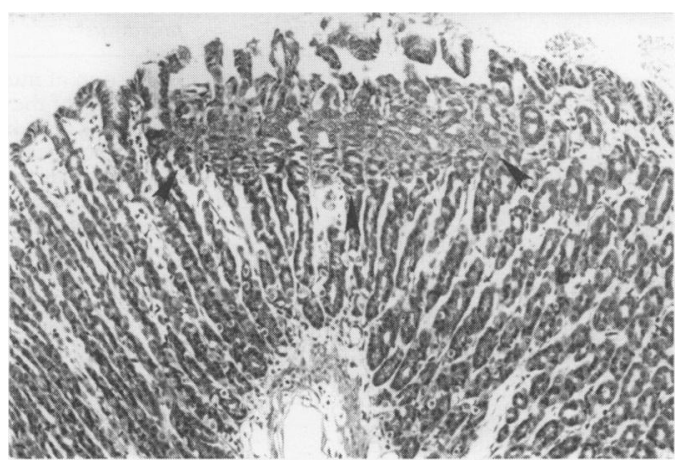

Figure 2: The apex of a corpus mucosal fold from a fasted rat that received oral indomethacin five minutes earlier. There is a well defined area of clumping and distortion of the gastric glands in the upper half of the mucosa (arrowheads). The overlying surface epithelium is fragmented and has separated from the underlying lamina propria. Haematoxylin and eosin, original magnification $\times 100$.

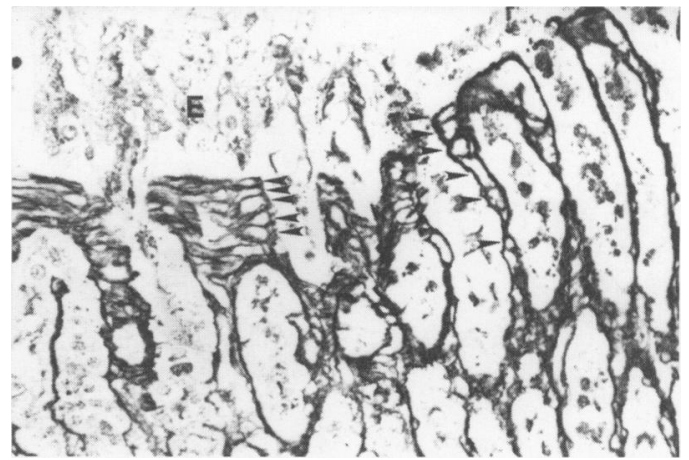

Figure 3: Reticulin stain of the edge of the lesion shown in Figure 2. There is buckling and flattening of the mucosa on the left hand of the field while the taller mucosa on the right is normal. The compressed and normal capillaries are depicted in the abnormal and normal areas respectively (arrowheads). The outline of extruded epithelium is outlined $(E)$. Reticulin stain, original magnification $\times 250$.

cells and greater intravascular fibrin deposition within the surface microvasculature (Fig 4; Table I). Non-perfused red blood cells within surface capillaries depicted a picture of vascular congestion. Neutrophils were seen adhering to the luminal aspect of vascular endothelial cells and within the lamina propria. At 30 minutes after dosing, the abnormality developed into coagulative necrosis of the superficial third of the mucosa with further neutrophil infiltration and mucosal contraction (Figs 5 and 6).

Overall, the histological sequence emerging from these findings was one of a focal, rapid, and progressive mucosal contraction that did not, at least initially, depend on the presence of infiltrating neutrophils.

The antrum was histologically normal in all indomethacin dosed rats. None of the sections taken from the control rats showed any histological abnormality in either the corpus or the antrum. Table II summarises the histological findings identified in the corpus of fasted rats.

TABLE I Neutrophil infiltration and measurement of the mucosal thickness of corpus and antral lesions induced by indomethacin $30 \mathrm{mg} / \mathrm{kg}$ and vehicle in fasted and fastedrefed rats at various times after dosing

\begin{tabular}{|c|c|c|}
\hline & $\begin{array}{l}\text { Lesion/normal } \\
\text { mucosal thickness } \\
\text { ratio }\end{array}$ & $\begin{array}{l}\text { Neutrophils/ } \\
\text { lesion } † \ddagger\end{array}$ \\
\hline \multicolumn{3}{|l|}{ Corpus lesions in fasted rats } \\
\hline $5 \mathrm{~min}$ after indomethacin & $0.86(0.07)$ & 0 \\
\hline $15 \mathrm{~min}$ after indomethacin & $0.75(0.03)$ & $1 \cdot 6(0 \cdot 8)$ \\
\hline $30 \mathrm{~min}$ after indomethacin & $0.60(0.06)$ & $1 \cdot 1(0 \cdot 3)$ \\
\hline 5 min after vehicle & - & 0 \\
\hline $15 \mathrm{~min}$ after vehicle & - & 0 \\
\hline 30 min after vehicle & - & 0 \\
\hline \multicolumn{3}{|l|}{ Antral lesions in fasted-refed rats } \\
\hline $1 \mathrm{~h}$ after indomethacin & $0.69(0.06)$ & 0 \\
\hline $2 \mathrm{~h}$ after indomethacin & $0.50(0.05)$ & $0.4(0.3)$ \\
\hline $4 \mathrm{~h}$ after indomethacin & $0.60(0.02)$ & $49(22)$ \\
\hline $1 \mathrm{~h}$ after vehicle & - & 0 \\
\hline $2 \mathrm{~h}$ after vehicle & - & 0 \\
\hline $4 \mathrm{~h}$ after vehicle & - & 0 \\
\hline
\end{tabular}

^Using a calibrated eyepiece graticule attached to a microscope, the mucosal thickness of lesions was measured and expressed as the ratio lesion mucosal thickness/mucosal thickness of adjacent normal mucosa. The mean (SEM) from each rat was determined from measurements of two to six lesions per rat. The corresponding ratio for the normal mucosa of control rats is considered to be $1 \cdot 0$. $†$ Neutrophils within lesions (one to six lesions per rat) were counted using the ANS lesions (one to six lesions per rat) were counted using the ANS the mucosal folds of vehicle control rats. 


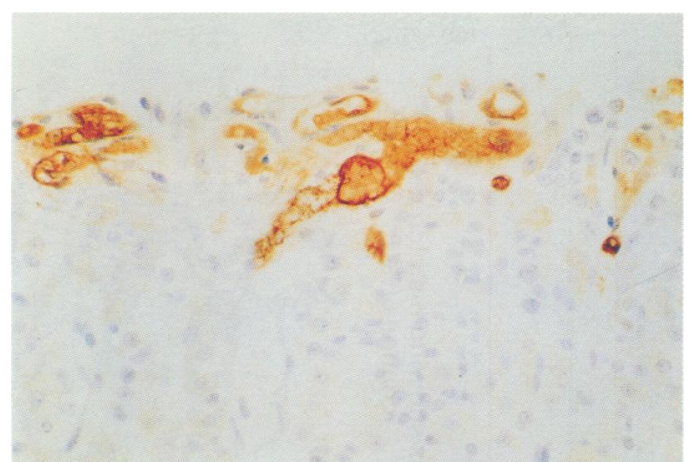

Figure 4: Plugging of surface capillaries with fibrin thrombi 15 minutes after indomethacin in the fasted rat. The surface epithelium is absent. Immunohistochemical stain for fibrinogen, original magnification $\times 250$.

Gastric lesions in fasted-refed rats

Subcutaneous indomethacin induced time dependent formation of macroscopic antral haemorrhagic spots that were mainly seen six hours after dosing with indomethacin and to a lesser extent at two hours (Fig 7). At one hour the antral mucosa showed poorly defined areas of pallor rather than discrete haemorrhagic lesions. No corpus lesions were seen in any of the fasted-refed indomethacin or vehicle control animals. None of the control rats showed any visible signs of injury to the antrum. The stomachs of all indomethacin and control rats were severely distended with semidigested food.

In four of five rats at one hour, the sides of antral mucosal folds showed histological foci of full thickness mucosal coagulative necrosis on the apices and along the sides of mucosal folds (Fig 8). Lesions showing a lesser degree of necrosis had the features of a contracted mucosa having staining characteristics similar to the corpus lesions seen in the fasted rats that is, a picture of mucosal contraction as seen in the reticulin and actin stained sections that featured capillary distortion and contractile cell thickening and shortening with a reduction of mucosal thickness (Table I).

A similar spectrum of antral mucosal injury was noted at two hours except that occasional neutrophils were identified within degenerate

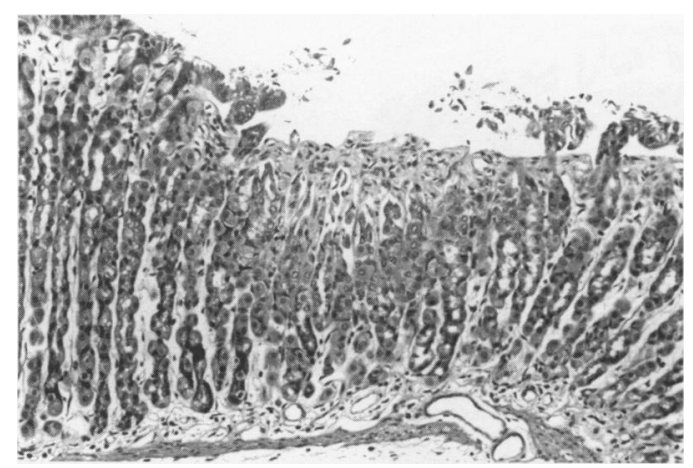

Figure 5: $A$ corpus erosion showing epithelial loss and coagulative necrosis of the upper half of the mucosa 30 minutes after oral indomethacin. The lesion shows mucosal compression and reticulin staining of the same lesion confirmed an intact surface capillary network without loss of the lamina propria. Only one neutrophil was identified in this lesion by ANS immunostaining. Haematoxylin and eosin, original magnification $\times 100$.

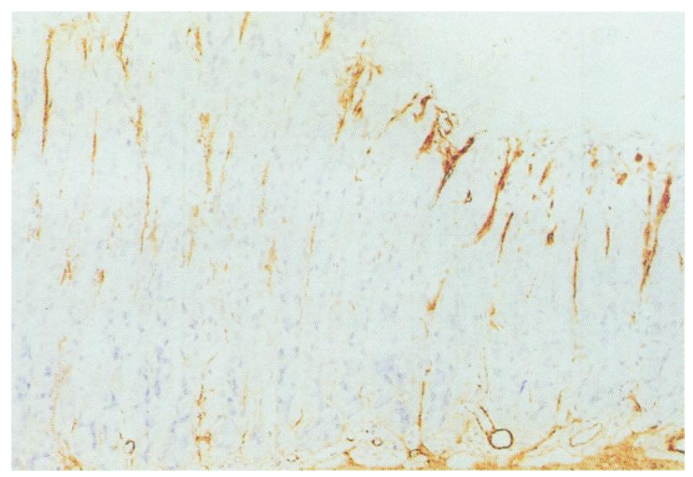

Figure 6: Actin stain of the lesion shown in Figure 5 showing darker staining of the mucosal contractile cells on the right. The cells are also thickened in comparison to the ones within the mucosa on the left. Actin immunostaining, original magnification $\times 100$.

mucosa. Vascular fibrin staining was a major feature within the mucosa but large underlying submucosal vessels also contained occlusive fibrin thrombi.

At four hours, numerous neutrophils were seen infiltrating the lamina propria of the mucosa and adherent to the endothelium of large submucosal vessels. Most neutrophils, however, were found as a large, dense band of cells along the base of the muscularis mucosae (Fig 9; Table I).

In each of the one, two, and four hour groups dosed with subcutaneous indomethacin, four of five rats showed histological corpus lesions that consisted of areas of focal mucosal contraction identical to those seen in the fasted rats five minutes after indomethacin dosing. None of the fasted-refed control rats showed histological abnormalities. Table II summarises the macroscopic and histological changes described in the fasted-refed rats.

\section{Discussion}

Using perfusion fixation of tissues and histological and immunohistochemical techniques, we have described a sequence of progressive

TABLE II Summary of the histological changes seen in the corpus and antrum of fasted and fasted-refed rats at various times after dosing with indomethacin

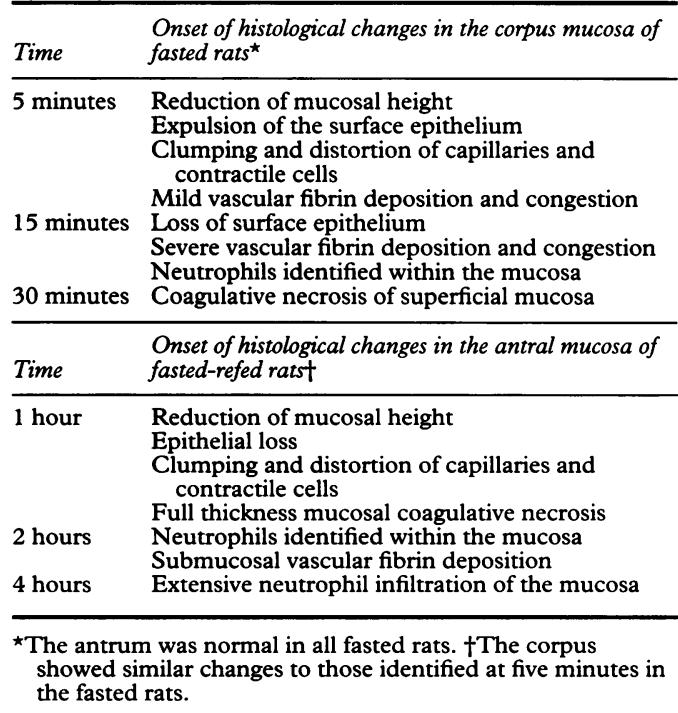




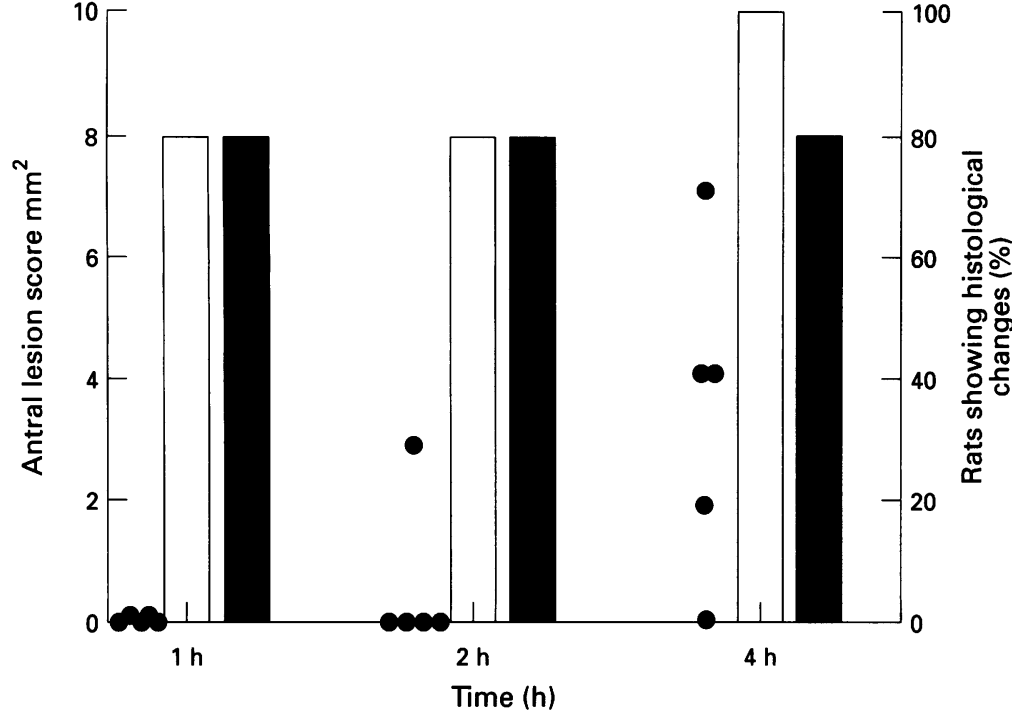

Figure 7: The extent of visible and histological injury induced by subcutaneous indomethacin $30 \mathrm{mg} / \mathrm{kg}$ in the corpus and antrum of fasted-refed rats at one, two, and four hours after dosing. Filled circles=antral ulcer score for individual indomethacin dosed rats $(n=5$ per group). Open bar=percentage of rats showing antral histological changes. Solid bar=percentage of rats showing corpus histological changes. The corpus was macroscopically normal in all indomethacin dosed rats. None of the corresponding vehicle control rats showed any macroscopic or histological abnormality to either the corpus or antrum.

NSAID induced injury to the gastric mucosa of the corpus and antrum of fasted and fastedrefed rats, respectively. We have identified early phases of injury that did not involve infiltration of neutrophils in both models of gastric mucosal injury.

As early as five minutes after an oral dose of indomethacin to fasted rats, at a time when there were no visible erosions to the naked eye, there were significant architectural changes to superficial mucosal glandular, vascular, and contractile cells as well as capillary fibrin deposition. The nature of the architectural changes, in particular, suggested that the mucosa had undergone contraction - that is, a reduction in thickness. Whether this contraction was a consequence of activation of either mucosal contractile cells (smooth muscle cells and myofibroblasts) or the muscularis mucosa, or both, is not clear at present although it is worth mentioning that both constitute an elaborate contractile network within the superficial bowel wall. It is possible that contraction of the muscularis

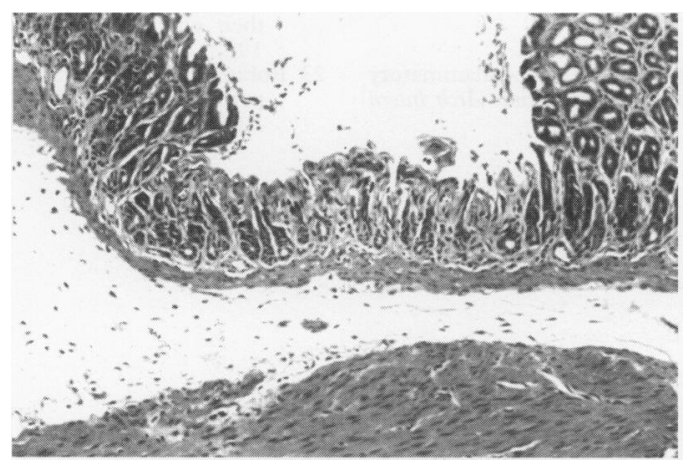

Figure 8: An antral ulcer showing coagulative necrosis of a contracted mucosa one hour after indomethacin in a fastedrefed rat. Some glandular remnants are still visible. Not a single neutrophil is seen and nor were they identified in the ANS stained section. Haematoxylin and eosin, original magnification $\times 100$.

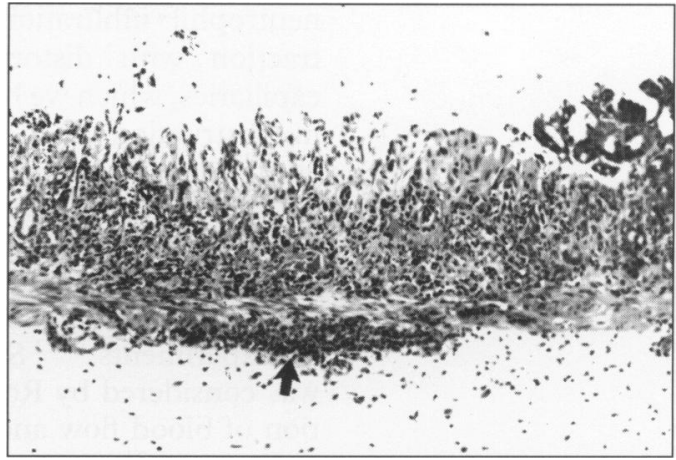

Figure 9: An antral ulcer showing localised coagulative necrosis of the mucosa four hours after indomethacin in a fasted-refed rat. Numerous neutrophils are infiltrating the mucosa and forming $a$ band of cells just beneath the muscularis mucosae (arrow). Haematoxylin and eosin, original magnification $\times 100$.

mucosae could explain the mucosal congestion as veins and arteries pass through this layer. Also, a nervous influence cannot be ruled out as the rat oesophageal muscularis mucosae is known to be innervated by motor cholinergic ${ }^{16}$ and spasmolytic $\beta_{3}$-adrenoceptor sympathetic nerves. ${ }^{17}$

The fact that the surface epithelium and superficial glands appeared to be physically exfoliated by a compression of the underlying structures tends to support the notion of a spasmodic event early in the pathogenic process. The surface vessels having been damaged by this deformation showed progressively more deposition of fibrin 10 minutes later. Only at this time did neutrophils infiltrate the mucosa and even then in very few numbers that did not appear to be commensurate with the degree of mucosal damage if these leucocytes were the primary cause of ulceration. Both their delayed arrival and the low number of neutrophils appearing are findings that strongly suggest that the latter are a secondary response to tissue injury and are not involved in the primary initiating events of NSAID injury in this model. The antral lesions arising in fasted-refed rats also showed a similar sequence of histological events in which the mucosa underwent contraction and necrotic phases of injury that were first seen one hour after dosing with indomethacin. Importantly, the necrotic phase was neutrophil-free: not a single neutrophil was identified within the ANS stained sections at this time. Neutrophils were only identified at two hours after dosing, which supports a secondary role for these circulating cells that arrive in response to tissue necrosis.

We hypothesise that the severe vascular deformation and vascular fibrin deposition identified in each model of gastric injury impedes mucosal blood flow and result in ischaemic damage. That the affected capillaries alşo showed congestion of intravascular red blood cells (despite perfusion fixation at mean arterial pressure) supports the idea that some obstruction to blood flow had occurred at some stage.

We have previously described a similar sequence of histological events in the small intestine of indomethacin dosed rats. Prior to 
neutrophil infiltration, villi underwent contraction with distortion of the superficial capillaries, which we hypothesised would result in obstruction of blood flow and ischaemic injury due to activation of contractile cells within the villous core. ${ }^{12-14}$

Earlier morphological studies have described the superficial gastric microvasculature as being an important target of NSAID toxicity in rat experiments. ${ }^{18-23}$ Such microvascular injury was considered by Robins to lead to interruption of blood flow and ischaemic infarction of the mucosa. ${ }^{23}$

We examined neutrophil infiltration in relation to injury of mucosal structures, in particular, to answer the question as to whether neutrophil infiltration is the first abnormality to be detected. Our data show that mucosal contraction and occlusive vasculopathy occur prior to neutrophil infiltration and support the recent findings of others who have shown NSAID gastric injury to be neutrophil independent in the neutropenic rat. ${ }^{10} 11 \mathrm{~A}$ histological study published in abstract form by Whiteley et al described indomethacin induced mucosal injury to the rat corpus that was not related to either a significant increase in ANS positive cells within the mucosa or a fall in circulating neutrophils at the onset of injury over control rats. ${ }^{9}$

It could be argued that the perfusion-fixation technique had removed the earliest intravascular neutrophils from the vascular tree. However, the first neutrophils to be identified within the corpus at 15 minutes and in the antrum at four hours were noted to be adherent to the luminal aspect of the vascular endothelium at these times. Vascular perfusion fixation does not, therefore, flush out adherent neutrophils from injured mucosa.

In summary, the experiments reported in this paper have described the sequence of histological changes in two models of NSAID ulceration in the rat. In each case, we have identified an early phase of injury that involves mucosal contraction and vascular fibrin deposition but does not involve neutrophil infiltration initially. These findings do not support a primary role for neutrophils in NSAID gastropathy.

This project was supported by Glaxo Wellcome Research and Development, Stevenage, UK.

1 Roth SH, Bennett RE. Non-steroidal anti-inflammatory drug gastropathy: Recognition and response. Arch Intern Med 1987; 147: 2093-100.
2 Scarpignato C. Non-steroidal anti-inflammatory drugs: How do they damage gastroduodenal mucosa? Dig Dis 1995; 13 (suppl 1): 9-39.

3 Wallace JL, Keenan CM, Neil Granger D. Gastric ulceration induced by non-steroidal anti-inflammatory drugs is a neutrophil dependent process. Am $\mathcal{F}$ Physio 1990; 259: G462-7.

4 Wallace JL, Arfors KE, Webb McKnight G. A monoclonal antibody against the CD18 leukocyte adhesion molecule prevents indomethacin-induced gastric damage in the rabbit. Gastroenterology 1991; 100: 878-83.

5 Lee M, Aldred K, Lee E, Feldman M. Aspirin-induced gastric mucosal injury is a neutrophil-dependent process in rats. Am f Physiol 1992; 263: G920-6.

6 Wallace JL, McKnight W, Miyasaka M, Tamatani T, Paulson J, Anderson DC, et al. Role of endothelial adhesion molecules in NSAID-induced gastric mucosal injury. Am $\mathcal{F}$ Physiol 1993; 265: G993-8.

7 Wallace JL. Non-steroidal anti-inflammatory drug gastropathy and cytoprotection: Pathogenesis and mechanisms re-examined. Scand $¥$ Gastroenterol 1992; 27 (suppl 192): 3-8.

8 Arndt H, Palitzsch K-D, Anderson DC, Rusche J, Grisham MB, Granger DN. Leucocyte-endothelial cell adhesion in a model of intestinal inflammation. Gut 1995; 37: 374-9.

9 Whiteley LO, Erickson A, Smith B. NSAID-induced acute gastric injury in rats: Where are the neutrophils? A histological study. Gastroenterology 1995; 108: A258.

10 Trevethick MA, Bahl AK, Clayton NM, Strong P, Sanjar S, Harman IW. Neutrophil infiltration does not contribute to the ulcerogenic effects of indomethacin in the rat gastric mucosa. Agents Actions 1994; 43: 39-43.

11 Melarange R, Gentry C, Toseland N, Smith PH, Fuller J. Neutropenia does not prevent etodolac- or indomethacininduced gastrointestinal damage in the rat. Dig Dis $\mathrm{Sci}$ 1995; 40: 2694-703.

12 Anthony A, Dhillon AP, Nygard G, Hudson M, Piasecki C, Strong $\mathrm{P}$, et al. Early histological features of smal intestinal injury induced by indomethacin. Alimen Pharmacol Ther 1992; 7: 29-40.

13 Nygard G, Anthony A, Piasecki C, Trevethick MA, Hudson $M$, Dhillon AP, et al. Acute indomethacininduced injury in the rat: early morphological and biochemical changes. Gastroenterology 1994; 106: 567-75.

14 Anthony A, Thrasivoulou C, Dhillon AP, Pounder RE, Wakefield AJ. Pre-ulcerative villous contraction and microvascular occlusion induced by indomethacin in the rat jejunum: A detailed morphological study. Aliment Pharmacol Ther 1995; 9: 605-13.

15 Satoh H, Inada I, Hirate T, Maki Y. Indomethacin produces antral ulceration in the refed rat. Gastroenterology 1981 ; 81: 719-25.

16 Bieger $D$, Triggle C. Pharmacological properties of mechanical responses of the rat oesophageal muscularis mucosae to vagal and field stimulation. Br $\mathcal{f}$ Pharmacol 1985; 84: 93-106.

17 de Boer REP, Brouwer F, Zaagsma J. Noradrenalin-induced relaxation of rat oesophageal muscularis mucosae: mediation solely by innervated $\beta_{3}$-adrenoceptors. $\mathrm{Br}$ Pharmacol 1995; 116: 1945-7

18 Rainsford $\mathrm{KD}$. Electron microscopic observations on the effects of orally administered aspirin and aspirinbicarbonate mixtures on the development of gastric mucosal damage in the rat. Gut 1975; 16: 514-27.

19 Rainsford KD, Fox SA, Osborne DJ. Comparative effects of some anti-inflammatory drugs on the ultrastructural integrity and prostaglandin levels in the rat gastric integrity and prostaglandin levels in the rat gastric mucosa: Relationship to drug uptake.

20 Tarnawski A, Stachura J, Gergely H, Hollander D. Gastric microcircular endothelium: a major target for aspirininduced injury and arachidonic acid protection. An ultrastructural analysis in the rat. Eur $\mathcal{F}$ Clin Invest 1990 20: 432-40.

21 Hingson DJ, Ito $S$. Effects of aspirin and related compounds on the fine structure of mouse gastric mucosa. Gastroenterology 1971; 61: 156-77.

22 Rainsford KD, Willis C. Relationship of gastric mucosal damage induced in pigs by anti-inflammatory drugs to their effects on prostaglandin production. Dig Dis $S c i$ 1982; 27: 624-35.

23 Robins PG. Ultrastructural observations on the pathogenesis of aspirin-induced gastric erosions. $B r \mathcal{F}$ Exp
Pathol 1980; 61: 497-504. 